\title{
Synthesis route and optical characterization of CdS:Mn/polyvinyl alcohol nanocomposite
}

\author{
V.I. Fediv', G.Yu. Rudko ${ }^{1}$, A.I. Savchuk ${ }^{3}$, E.G. Gule ${ }^{2}$, A.G. Voloshchuk ${ }^{3}$ \\ ${ }^{1}$ Bukovyna State Medical University, \\ 42, Kobylyanska str., 58012 Chernivtsi, e-mail: vfediv@ukr.net \\ ${ }^{2} V$. Lashkaryov Institute of Semiconductor Physics, NAS of Ukraine, \\ 45, prosp. Nauky,03028Kyiv,e-mail: rudko@isp.kiev.ua \\ ${ }^{3}$ Chernivtsi National University, 2, Kotsyubynsky str., 58012 Chernivtsi, Ukraine
}

\begin{abstract}
CdS nanoparticles doped with Mn have been synthesized in aqueous solution by using polyvinyl alcohol (PVA) as a capping reagent. Influence of the polymer concentration on the size and concentration of the nanoparticles was studied using transmission electron microscopy and optical absorption. Incorporation of $\mathrm{Mn}^{2+}$ ions into $\mathrm{CdS}$ nanoparticles was confirmed by photoluminescence (PL) intensity increase and band shift with Mn concentration. The PL studies proved that localization of $\mathrm{Mn}$ ions (inside the nanoparticles or on the surface) depends on the sequence of synthesis steps. The bleaching of the low-energy PL band of the low-doped CdS:Mn nanoparticles by surface modification with a $\mathrm{Cd}(\mathrm{OH})_{2}$ layer proves suppression of the emission related to surface states.
\end{abstract}

Keywords: nanoparticle, semimagnetic semiconductor, polyvinyl alcohol, photoluminescence.

Manuscript received 02.03.12; revised version received 21.03.12; accepted for publication 27.03.12; published online 30.05.12.

\section{Introduction}

The synthesis and study of nanostructured materials have become a major area of interdisciplinary studies over the past 20 years. In particular, the hybrid polymersemiconductor materials are becoming increasingly attractive because of a large number of applications, including photonics, optoelectronics, and biotechnology. The reason is that the polymer matrices exhibit extraordinary advantages such as film formability, good transparency, solubility, on the one hand, and provide wide possibilities to control the size and morphology of nanoparticles during their growth, on the other hand $[1,2]$.

$\mathrm{CdS}$ is an important direct band gap II-VI semiconductor and a low cost material. Fabrication and characterization of $\mathrm{CdS}$ nanoparticles embedded into polymer matrices have been extensively studied (see, e.g. [3-6]). In their turn, semiconductor nanoparticles can serve as a unique host for doping with optically active impurities due to their size-dependent band gap and host-impurity electronic interactions. The doping of semiconductor nanoparticles with magnetic ions (e.g., with $\mathrm{Mn}$ ) plays a dual role in controlling their optical properties: i) the band gap of the compound varies with the concentration of manganese ions, ii) the $3 d$ levels of transition-metal ions are located in the band gap and, thus, $d-d$ transitions alter the spectral characteristics.

During the past decades, the $\mathrm{Mn}^{2+}$-doped $\mathrm{CdS}$ nanoparticles have been fabricated by chemical synthesis methods such as co-precipitation, reverse micelles, hydrothermal and organometallic procedures [7-10]. However, incorporation of dopants into semiconductor nanocrystals by chemical methods remains a practical challenge since the impurities generally tend to be excluded from nanoparticles during crystal growth. Dopant exclusion from a seed has been suggested to be a general phenomenon $[11,12]$ and the modes of dopant binding to crystallite surfaces have been investigated in the context of impurity incorporation $[13,14]$. 
In this work, the synthesis and characterization of colloidal solution of $\mathrm{Mn}^{2+}$-doped $\mathrm{CdS}$ nanoparticles capped with PVA and CdS:Mn nanoparticles/PVA solid nanocomposite films is reported. These materials have been studied using optical absorption, TEM and photoluminescence spectroscopy. We focused on the analysis of the influence of PVA concentration on the size and concentration of colloidal nanoparticles that has not been discussed before. We demonstrated that the proposed synthesis method provides efficient incorporation of $\mathrm{Mn}$ impurity into $\mathrm{CdS}$ nanoparticles contrary to the preceding reports for the same nanosystem [10]. We also applied termination of CdS:Mn nanoparticle surface with $\mathrm{Cd}(\mathrm{OH})_{2}$ shell and achieved the passivation of surface defects. As a consequence, we achieved variation of optical properties that was not observed previously [9].

\section{Experimental}

\subsection{Samples preparation}

CdS:Mn nanoparticles were synthesized in the multicomponent system $\mathrm{CdCl}_{2}-\mathrm{MnCl}_{2}-\mathrm{Na}_{2} \mathrm{~S}-\mathrm{H}_{2} \mathrm{O}$ capping molecules at room temperature by coprecipitation method described in [15]. Polyvinyl alcohol (PVA) was used as a capping agent. It has a molecular weight close to 50,000, and a degree of hydrolysis from 99.0 to $99.5 \%$. The synthesis conditions, i.e. concentrations of salt solutions of $\mathrm{CdCl}_{2}, \mathrm{MnCl}_{2}$, $\mathrm{Na}_{2} \mathrm{~S}$ and the $\mathrm{pH}$ value, were adjusted to prevent precipitation of $\mathrm{MnS}, \mathrm{Mn}(\mathrm{OH})_{2}$ and $\mathrm{Cd}(\mathrm{OH})_{2}$. Analysis of the probability of chemical reactions in the multicomponent system $\mathrm{Cd}^{2+}-\mathrm{Mn}^{2+}-\mathrm{S}^{2-}-\mathrm{H}_{2} \mathrm{O}$ for the chosen synthesis conditions shows that the optimal ranges of molar concentrations of precursors and the $\mathrm{pH}$ value are $10^{-4} \ldots 10^{-2} \mathrm{~mol} / \mathrm{l}$ and 3 to 5 , respectively. In order to keep the system in the focusing regime (i.e., to ensure the formation of nearly monodisperse nanocrystals) for a sufficiently long time, it is necessary to perform additional slow injections of precursors during the growth, effectively keeping the concentration of free species in a solution above a critical threshold. All steps of the synthesis were performed under ambient conditions. All chemicals used in the present work were analytical-grade and were used without any further purification.

In the work, the properties of CdS:Mn nanoparticles were governed by the polymer concentration, $\left[\mathrm{CdCl}_{2}\right]$ : $\left[\mathrm{MnCl}_{2}\right]$ molar ratio, $\mathrm{pH}$ value and the sequence of precursor addition. The sulfide concentration was stoichiometric. The synthesis time was about 1 to $2 \mathrm{~h}$.

The method yielded colloidal solutions of CdS:Mn nanoparticles that were further used to form solid nanocomposite films. The nanocomposite films of the CdS:Mn nanoparticles/PVA were formed by adsorptive dessication method. For this purpose, the colloid solution was transferred to glass Petri dishes which were placed in a pressure-tight vessel that contained an absorbent. The temperature of drying was $20^{\circ} \mathrm{C}$.

Incorporation of paramagnetic impurity into nanoparticles was done using the adsorptive doping method that implies several steps of synthesis. The first step is preparation of PVA solution containing $\mathrm{Mn}^{2+}$ ions. The concentration of $\mathrm{Mn}^{2+}$ precursor within the range $\left(10^{-3} \ldots 10^{-1} \mathrm{~mol} / \mathrm{l}\right)$ is an important parameter that influences the process of doped nanoparticles growth. During the second step, $\mathrm{Cd}^{2+}$ and $\mathrm{S}^{2-}$ precursors are added to the above solution, and seeds of $\mathrm{CdS}$ nanoparticles are formed. The $\mathrm{Mn}^{2+}$ ions are adsorbed on the surface of the seeds (it should be stressed here that the chosen synthesis conditions prevent $\mathrm{MnS}$ formation). All the following steps of synthesis are the alternative additions of the precursors of $\mathrm{Cd}^{2+}$ and $\mathrm{S}^{2-}$. During every growth step, $\mathrm{Mn}^{2+}$ ions are adsorbed on the surface of the increased particle and, thus, the dopant was burried under the surface of nanoparticle during the following steps of growth. The parameters of the synthesis of colloidal solution of CdS:Mn nanoparticles were: synthesis time $-1 \mathrm{~h}$, temperature $-293 \mathrm{~K}$, solvent - water, concentration of $\mathrm{Cd}^{2+}$ and $\mathrm{S}^{2-}$ precursors $3 \times 10^{-3} \mathrm{M}$, concentration of $\mathrm{Mn}^{2+}-3 \times 10^{-3} \mathrm{M}$.

\subsection{Characterization}

Optical absorption measurements were carried out using the grating monochromator for the $200-2200 \mathrm{~nm}$ wavelengths range with UV and visible light sources. The optical density of colloidal solution was measured using the pure polymer solution for comparison.

In the course of measurements, the temperature was $300 \mathrm{~K}$. The photoluminescence (PL) spectra were measured using the grating monochromator MDR-23 and LED source NS-375L-5RLO $(\lambda=375 \mathrm{~nm})$ for excitation. The spectral resolution was $0.5 \mathrm{~nm}$, the temperature of measurements varied from 4.2 to $300 \mathrm{~K}$.

Transmission electron microscopy (TEM) images were obtained with an electron microscope JEOL JEM$100 \mathrm{SX}$ operating at an accelerating voltage of $100 \mathrm{kV}$.

\section{Results and discussion}

A colloidal solution of CdS:Mn nanoparticles can be stabilized by the adsorption of polymer molecules onto the nanoparticles. Efficiency of stabilizing action of polymers can be determined by the change in the threshold of coagulation that is registered by optical density measurements. An important consequence of stabilizing with polymer is the formation of nanoparticles of certain sizes and concentrations.

The minimal number of adsorbed polymer molecules that inhibit the further growth of a nanoparticle, and, thus, define its size, depends on the concentration of polymer in a solution as well as on the character of an interaction of macromolecules in the adsorbed layer. Fig. 1 shows the optical 
absorption results illustrating the effect of the polymer concentration on the spectral dependences of optical density of the colloidal solutions of CdS:Mn nanoparticles. Note that each colloid was synthesized using the maximum possible concentrations of precursos $\left(\mathrm{CdCl}_{2}, \mathrm{Na}_{2} \mathrm{~S}\right)$ that do not exceed the coagulation threshold. Two characteristics of these spectra are to be taken into consideration - the energy position of the peak in the absorption spectrum and relative value of the optical density. The position of the peak characterises the average diameter of nanoparticles in the solution, the optical density reflects the concentration of nanoparticles.

It is seen from Fig. 1 that variation of PVA concentration over the range 1 up to $10 \mathrm{wt} \%$ does not influence the position of the absorption peak $(h v \approx$ $3 \mathrm{eV}$ ). Therefore, one can conclude that the size of the CdS:Mn nanoparticles does not change within this range of PVA concentrations.

Another important factor for stability of a colloidal system is the concentration of nanocrystals in it. The latter is related to the absolute value of optical density of colloidal solution. It is seen from Fig. 1 that increasing of the concentration of polymer in the solution leads to non-monotonous changes in the optical density: optical density at first grows with the increase of PVA concentration and then decreases. These changes are indicative of nanoparticles concentration variation. The highest peak in the absorption spectrum is observed when the concentration of polymer is equal to $5 \mathrm{wt} . \%$, i.e. at this concentration of polymer the maximal content of nanoparticles in the solution is obtained. To elucidate the reasons of the observed non-monotonous behaviour within the range $1 \ldots 10 \mathrm{wt} . \%$ of PVA concentration, one has to analyze two factors: i) how does the number of PVA macromolecules, that are directly attached to the surface of a CdS:Mn nanocrystal, change with PVA concentration, ii) the role of the PVA molecules in the solution beyond the first adsorbed layer.

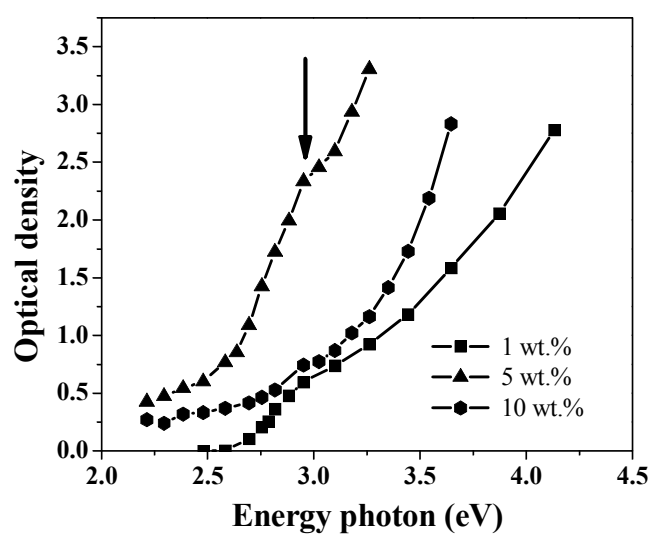

Fig. 1. Spectral dependence of the optical density of colloidal CdS:Mn nanocrystals in the PVA matrix.
As has been demonstrated using the nuclear magnetic resonance method [16], in an aqueous medium the elementary segment of PVA coordinates around itself two molecules of water and can occupy an area of $30 \AA^{2}$ on the surface of a colloidal particle. Taking into account this fact as well as the experimental results on the adsorption of PVA on AgI and $\mathrm{Sb}_{2} \mathrm{~S}_{3}$ colloidal particles the authors of [16] showed that the very first adsorbed layer comprises only $20-30 \%$ of the whole amount of polymer molecules adsorbed on the particle. In view of these two facts one can conclude that in our samples the majority of polymeric macromolecules in the solution do not contact with the surface of nanoparticles. Moreover, based on the results presented in Fig. 1 (that the average size of nanoparticles remains unchanged with the variation of PVA concentration) one can argue that the number of PVA macromolecules, that are directly attached to the surface of a CdS:Mn nanocrystal, does not change with the polymer concentration within the range 1 to 10 wt. $\%$.

Non-monotonous variation of the opical density of the colloid reflects the non-monotonous changes in the coagulation threshold. With the increase of PVA concentration the concentration of nanoparticles the colloid rises (compare curve 1 and 2 in Fig. 1) because the increased number of "free" polymer molecules provides better steric stabilization and prevents coagulation of nanoparticles. However, at even more high concentration of PVA (curve 3, Fig. 1) the steric stabilization is deteriorated by interactions between the macromolecules of polymer that lowers the coagulation threshold.

Analizing the energy position of the optical absorption peak (Fig. 1), one can estimate the average size of nanoparticles in the colloidal solution. It is seen that the absorption edge of CdS:Mn in the colloid is blueshifted as compared with the absorption edge of the bulk semiconductor $(2.42 \mathrm{eV}$ for bulk $\mathrm{CdS}$ ), thus proving formation of nanometer-sized $\mathrm{CdS}: \mathrm{Mn}$ particles in the samples. To find the average size of nanoparticles, we used both optical (Fig. 1) and transmission electron microscopy (TEM) (Fig. 2) studies.

We found the band gap $\left(E_{g}\right)$ of nanoparticles in our colloid samples from the absorption spectra using the method proposed in the paper [17]. The obtained band gap value $E_{g}$ is close to $2.95 \mathrm{eV}$. It is known that the band gap of nanoparticles is a function of a particle size. The latter can be estimated using either the tight binding approximation (TBA) or the effective mass approximation (EMA). According to [18], the experimental data agree well with the effective mass approximation for the diameters of the particles $>4 \mathrm{~nm}$, whereas TBA fits better for the particles with the diameters $<4 \mathrm{~nm}$. Murakoshi et al. [19] have reported that the experimental data for the dependence of band 
gap on the particle size agree well with EMA with a finite potential well even for smaller particle sizes. According to Brus [20], the energy band gap of the nanocrystals is

$$
E_{n p}=E_{g}+\frac{\hbar^{2} \pi^{2}}{2 r^{2}}\left(\frac{1}{m_{e}^{*}}+\frac{1}{m_{h}^{*}}\right)-\frac{1.8 e^{2}}{4 \pi \varepsilon_{0} \varepsilon_{r} r},
$$

where $E_{n p}$ is the band gap of the CdS:Mn nanoparticle. To get rough estimation of the size of $\mathrm{CdS}: \mathrm{Mn}$ nanoparticles in the composite, we used the values of the parameters for pure CdS. $E_{g}$ is the band gap of bulk CdS $(2.42 \mathrm{eV}), m_{e}^{*}-$ effective mass of an electron $\left(0.19 m_{e}\right.$ in $\left.\mathrm{CdS}\right), m_{h}^{*}$ - effective mass of a hole $\left(0.8 m_{e}\right.$ in $\left.\mathrm{CdS}\right), r$ - radius of the particle, $\varepsilon-$ dielectric constant equal to 5.7, and $\varepsilon_{0}$ is the permittivity of free space. Using the above equation, we have estimated the average particle size of PVAcapped CdS:Mn nanoparticles prepared at different polymer concentrations. The estimated radius of nanoparticles is $4 \mathrm{~nm}$.

We have controlled the sizes of nanoparticles in the composite by using TEM. Fig. 2 shows the TEM image of CdS:Mn nanocrystals in the PVA matrix. In these micrographs, the semiconductor nanocrystals appear as dark spots against a light background of the polymeric matrix. Grey regions that are also seen in the micrographs are attributed to some products of a reaction between PVA molecules and free ions. It is seen that the typical size of nanoparticles is about $10 \mathrm{~nm}$; similar sizes of nanoparticles were observed in the samples with PVA concentrations within the range $1 \ldots 10 \%$. This observation contradicts to the above calculations. The most probable source of this discrepancy is neglecting of the influence of $\mathrm{Mn}$ on the parameters of bulk material.

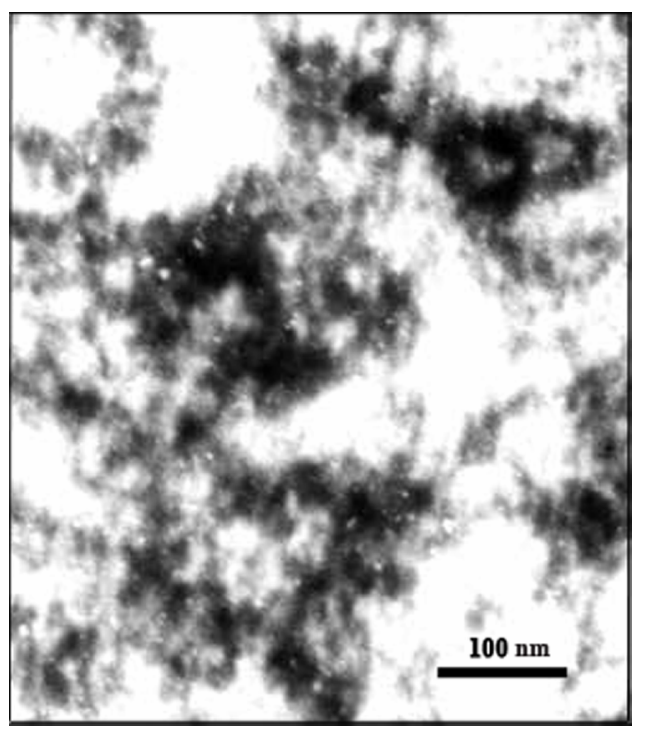

Fig. 2. TEM images of CdS:Mn nanocrystals in the PVA matrix.

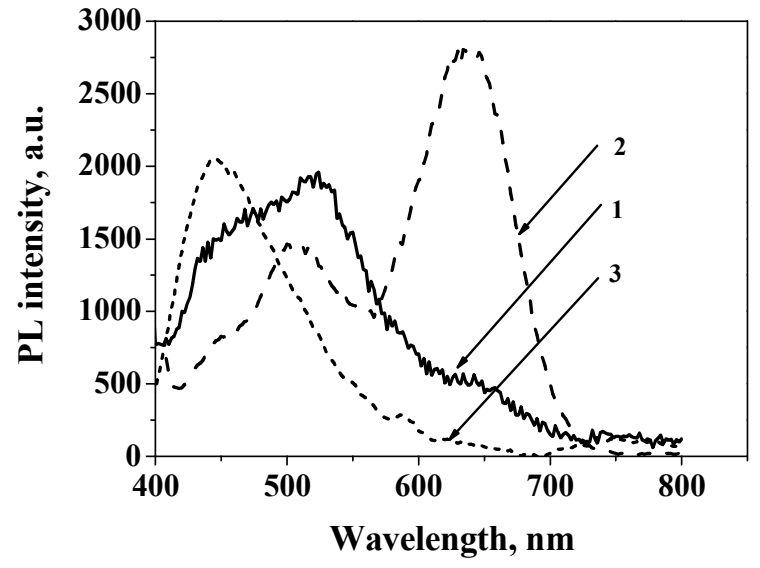

Fig. 3. PL spectra of $\mathrm{Mn}^{2+}$-doped $\mathrm{CdS}$ nanoparticles in PVA at $300 \mathrm{~K}(1), 4.2(2)$ and PL spectrum of pure PVA at $300 \mathrm{~K}$ (3). The ratio between $\mathrm{Cd}^{2+}$ and $\mathrm{Mn}^{2+}$ ions in the starting solution for synthesis is $1: 1$.

Solid films of nanocomposite CdS:Mn nanoparticles/PVA have also been studied by PL spectroscopy. Fig. 3 shows the PL spectra of $\mathrm{Mn}^{2+}$ doped $\mathrm{CdS}$ nanoparticles grown in $5 \mathrm{wt} \%$ PVA solution (curves 1 and 2). The measurements were carried out at $300 \mathrm{~K}$ (curve 1) and $4.2 \mathrm{~K}$ (curve 2). The PL spectrum of pure PVA measured at $4.2 \mathrm{~K}$ (curve 3) is shown for the comparison.

Three very wide spectral features are seen in the spectra of composite - two bands and a high-energy shoulder. By the comparison with the spectrum of pure PVA (curve 3), we ascribe the shoulder to the radiative transitions in the PVA matrix. Two bands are ascribed to the emission of nanoparticles. The high-energy band corresponds to the transitions involving shallow traps. The low-energy band is a complex feature; two types of emission overlap in this spectral region - one is ascribed to the transitions involving $d$-electrons of $\mathrm{Mn}^{2+}$ ions and another corresponds to the recombination via surface localized states in nanoparticles. Attribution of these bands will be discussed below.

All the nanoparticles-related bands are inhomogeneously broadened due to spatial averaging of the PL spectrum over the excitation area. As expected, the PL spectra are more intense at $4.2 \mathrm{~K}$ and a slight blue shift of the PL band at $\sim 500 \mathrm{~nm}$ with decreasing temperature is observed.

Fig. 4 shows PL spectra of $\mathrm{Mn}^{2+}$-doped $\mathrm{CdS}$ nanoparticles in PVA with various impurity concentrations: 1 - no doping, 2, 3 and $4-$ Mn-doped with the ratio of $\mathrm{Cd}^{2+}$ to $\mathrm{Mn}^{2+}$ in the starting solution for synthesis equal to $15: 1,5: 1$ and $1: 1$, respectively. It is seen that doping influences both spectral positions and intensities of the bands.

With the increase of Mn concentration, the highenergy band at first redshifts as compared to the corresponding peak in undoped $\mathrm{CdS}$ nanoparticles, while further addition of Mn causes its blueshift. This behavior 
closely resembles the variation of the band gap of bulk CdS:Mn crystals with Mn concentration [21], and thus it points to the successful incorporation of $\mathrm{Mn}$ into $\mathrm{CdS}$ nanoparticles.

The spectral position of the low-energy band does not depend on Mn content. Such independence of the dopant concentration (and, consequently, of the band gap value) is characteristic for the radiative transitions that do not involve delocalized charge carriers because the energy of the delocalized (shallowly trapped) charge carriers follows the energy shift of the conduction or valence bands. Thus, the low-energy band must be related either to deep defect levels or to surface localized states. Two facts support both of these assumptions. One of them is the increase of this band intensity with $\mathrm{Mn}$ doping. It was found (see the insert in Fig. 4) that the ratio of intensities high-energy to low-energy bands increases almost by 3 times with increasing $x$. This significant enhancement clearly indicates the role of $\mathrm{Mn}^{2+}$ doping. Therefore, we conclude that the intensity of low-energy band increases due to increasing contribution of the emission related to $\mathrm{Mn}$ ions. However, this mechanism could not be the only one responsible for the low-energy band. The second experimental fact is availability of this low-energy band in the spectra of undoped samples, which can be related to radiative transitions via surface localized states. Thus, based on the presence of low-energy band in the undoped samples - on the one hand, and on its intensity increase with doping - on the other hand, we conclude that this band is an overlap of the bands related to the localized surface states and to manganese impurity. We ascribe the Mn-related emission to the $d-d$ transitions of $\mathrm{Mn}^{2+}$ ions.

It is known that the $d$ - $d$-transitions in manganese ions are forbidden; however, in crystals they can be excited via the energy transfer from the host lattice to Mn ions [22-24]. The spectral position of the Mn $d-d$ band in nanocrystals depends on the surrounding crystalline field. Thus, the ${ }^{4} \mathrm{~T}_{1}-{ }^{6} \mathrm{~A}_{1}$ emission band of $\mathrm{Mn}^{2+}$ in CdS:Mn nanoparticles can be shifted to higher or lower energies as compared to $\mathrm{Mn}^{2+}$-related bands in bulk crystals. The transition energy for ${ }^{4} \mathrm{~T}_{1}-{ }^{6} \mathrm{~A}_{1}$ is observed to vary from 490 to $750 \mathrm{~nm}$ depending on the strength of crystal field $(D q)$ around the luminescent center. $\mathrm{Mn}^{2+}$ emission is also sensitive to the coordination geometry and number [25].

The strength of crystal field around a metal ion in a crystal lattice increases as the void containing the ion becomes smaller, with the increase of crystal field strength the energy for the above transition is predicted to decrease, hence the emission shifts to longer wavelengths according to Eq. [26]:

$D q=35 Z e / 4 R^{5}$,

where $R$ is the effective distance between metal ion and ligand, $Z e$ is the ion charge.
Emission from the first excited state to the ground state strongly depends on the host lattice. If the manganese ions are located in cation sites with an undistorted tetrahedral crystal field, excitation results in yellow emission at $588 \mathrm{~nm}(2.1 \mathrm{eV})$. If the tetrahedral crystal field is distorted, or if there are sites with octahedral symmetry, the crystal field is stronger and thus the states within the $3 d$ shell are significantly affected. Manganese ions in these lattice sites show different crystal field splittings of the $3 d$-orbital states, and therefore the emission bands are red-shifted [23, 25, 27], which agrees with our observations.

Luminescence of $\mathrm{Mn}^{2+}$ ions can serve as an instrument to study localization of these ions in the nanocomposite (whether they are incorporated into $\mathrm{CdS}$ nanoparticles or not). Fig. 5 shows the spectra of nanocomposites containing nanoparticles of three different types: CdS:Mn nanoparticles that were fabricated by adsorptive doping method (curve 1), CdS nanoparticles passivated by $\mathrm{Mn}$, i.e. the dopant was added to the solution when CdS nanoparticles have been already formed (curve 2), undoped CdS nanoparticles that were grown without $\mathrm{Mn}$ addition into the growth solution (curve 3 ). It is seen that the intensity ratio of the PL bands in the spectra of undoped and passivated nanoparticles are quite similar, thus passivation of $\mathrm{CdS}$ particles with $\mathrm{Mn}$ does not produce any additional radiative centers in nanoparticles. On the other hand, the relative intensities of the low-energy band in the spectra of Mn-passivated CdS and CdS:Mn nanoparticles are essentially different. This is a proof that adsorptive doping method provides efficient incorporation of $\mathrm{Mn}$ ions into the nanoparticles, while no doping occurs via diffusion of $\mathrm{Mn}$ from the surface towards the core of nanoparticle after passivation of nanoparticles (at the growth conditions used).

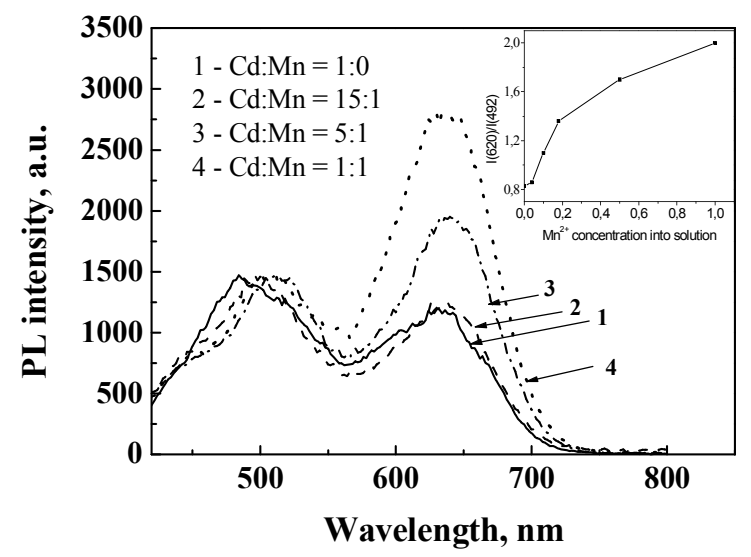

Fig. 4. Photoluminescence spectra of CdS:Mn nanoparticles in PVA matrix for samples with different $\mathrm{Cd}: \mathrm{Mn}$ ratio at $T=4.2 \mathrm{~K}$. Insert: Ratio of long to short wavelength bands intensities vs. the manganese concentration in solution. 


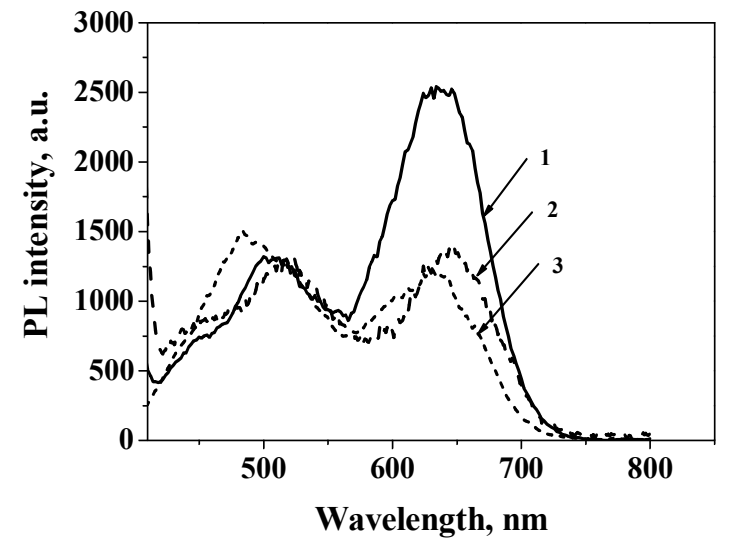

Fig. 5. Photoluminescence spectra of Mn-doped and undoped CdS nanoparticles in PVA matrix: Mn ions were not added (1); $\mathrm{Mn}$ ions were added to the solution after formation of nanoparticles (2); Mn ions were present in the growth solution during the synthesis (growth by the absorptive doping method) (3). The ratio between $\mathrm{Cd}^{2+}$ and $\mathrm{Mn}^{2+}$ ions in both latter cases was $1: 1 . T=4.2 \mathrm{~K}$

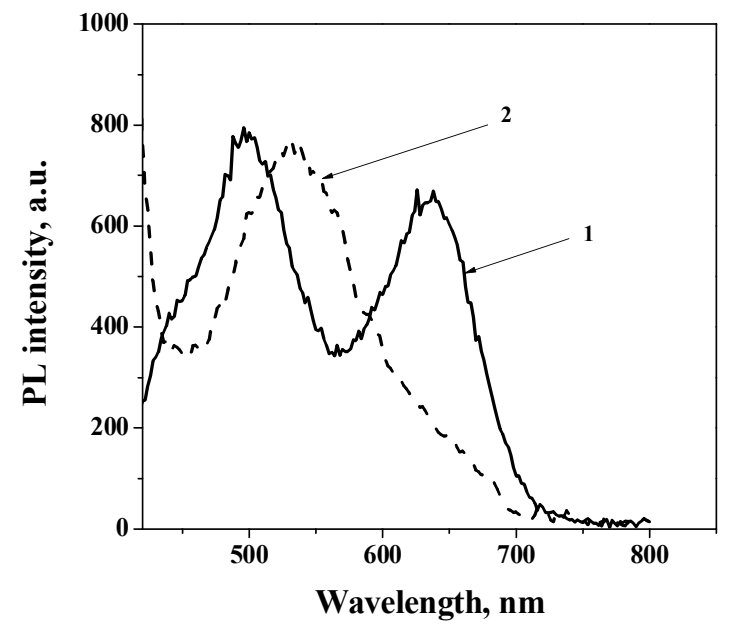

Fig. 6. PL spectra of CdS:Mn nanoparticles passivated by $\mathrm{Cd}(\mathrm{OH})_{2}$ shell: non-passivated CdS:Mn nanoparticles $(\mathrm{Cd}: \mathrm{Mn}$ $=15: 1)(1)$; CdS:Mn nanoparticles passivated by $\mathrm{Cd}(\mathrm{OH})_{2}$ shell (2). $T=4.2 \mathrm{~K}$

Observation of the low-energy band in the undoped nanoparticles (curve 1 in Fig. 4) points to the important contribution of surface trap states to the radiative processes in nanoparticles. The role of these states increases with decrease in the nanoparticle size. In a nanocrystal, surface atoms have some uncoordinated valences. To lower the total energy, these atoms rearrange their outer bonds (this effect is called surface reconstruction), thus introducing electronic states inside the band gap. In addition, the adsorption of some molecules on the surface can also introduce new states. All these states act as traps for electrons and holes. The nature of surface states depends mainly on the nanocrystal material and surface passivation. To elucidate the role of the surface states in our samples, we carried out passivation of nanoparticles with $\mathrm{Cd}(\mathrm{OH})_{2}$. Under the alkaline conditions in the solution during the synthesis, the $\mathrm{Cd}-\mathrm{OH}$ bond is formed on the CdS:Mn surface, therefore the number of the surface trap states diminishes, because the uncoordinated valences are passivated by the covering $\mathrm{Cd}-\mathrm{OH}$ shell.

The influence of passivation on PL of nanoparticles is illustrated in Fig. 6. Here, the curves 1 and 2 are the spectra of low-doped CdS:Mn nanoparticles with and without $\mathrm{Cd}(\mathrm{OH})_{2}$ shell, respectively. It is seen that the high-energy PL band of passivated nanoparticles is strongly redshifted and the intensity of low energy band drastically decreases.

In our samples (CdS:Mn nanoparticles/PVA solid nanocomposite films), the surface state transitions can be ascribed to the transition associated with $\mathrm{Cd}-\mathrm{O}$ complex [28] (Fig. 6, curve 1 as well as the spectra in Figs. 3 to 5). The decrease of the low energy peak intensity in the samples with nanoparticles passivated by $\mathrm{Cd}(\mathrm{OH})_{2}$ (Fig. 6, curve 2) may be caused by the coating applied, which restricts formation of the $\mathrm{Cd}-\mathrm{O}$ complex.

The red shift of the high-energy band (Fig. 6, curve 2) can be ascribed to covering the particles with the $\mathrm{Cd}(\mathrm{OH})_{2}$ shell. Contrary to the observations reported in $[9,29]$, we didn't observe essential increase of the high-energy band intensity.

\section{Conclusions}

Semiconductor CdS:Mn nanoparticles have been grown in PVA matrix by using the adsorptive doping method. Efficient incorporation of $\mathrm{Mn}^{2+}$ ions into $\mathrm{CdS}$ nanoparticles was achieved by optimization of the sequence of $\mathrm{Mn}^{2+}$ ions additions to the growth solution and proved by PL studies. It was shown that doping can be achieved only during the growth, and there is no diffusion of $\mathrm{Mn}$ ions from the surface of nanoparticles. When varying the PVA concentration within the range 1 to $10 \mathrm{wt} . \%$, the average size of nanoparticles remains unchanged, while their concentration varies. Their luminescence is strongly affected by modification of nanoparticle surface with $\mathrm{Cd}(\mathrm{OH})_{2}$. The nanocomposites synthesized are highly processable and can be fabricated in the form of films.

\section{Acknowledgement}

The work has been supported in part by the Ministry of Education and Science of Ukraine.

\section{References}

1. N. Tomczaka, D. Janczewski, M. Hana, G.J. Vancso, Designer polymer-quantum dot architectures // Progr. in Polymer Sci. 34, p. 393430 (2009). 
2. A.F.E. Hezinger, J. Teßmar, A. Gopferich, Polymer coating of quantum dots - a powerful tool toward diagnostics and sensorics // Europ. J. Pharmac. and Biopharmac. 68, p. 138-152 (2008).

3. Farid El-Tantawy, K.M. Abdel-Kader, F. Kaneko, Y.K. Sung, Physical properties of CdS-poly (vinyl alcohol) nanoconducting composite synthesized by organosol techniques and novel application potential // Europ. Polymer J. 40, p. 415-430 (2004).

4. J.C. Ferrer, A. Salinas-Castillo, J.L. Alonso, S. Fernandez de Avila, R. Mallavia, Synthesis and characterization of $\mathrm{CdS}$ nanocrystals stabilized in polyvinyl alcohol-sodium polyphosphate // Mater. Lett. 63(6-7) p. 638-640 (2009).

5. I.S. Elashmaw, N.A. Hakeema, M. Soliman Selim, Optimization and spectroscopic studies of CdS/poly(vinyl alcohol) nanocomposites // Mater. Chem. and Phys. 115(1), p. 132-135 (2009).

6. H. Wang, Z. Chen, P. Fang, S. Wang, Synthesis, characterization and optical properties of hybridized CdS-PVA nanocomposites // Mater. Chem. and Phys. 106, p. 443-446 (2007).

7. Q. Wang, Z. Xu, L. Yue and W. Chen, Characteristics and optical properties of $\mathrm{Cd}_{1-\mathrm{x}} \mathrm{Mn}_{\mathrm{x}} \mathrm{S}$ nanorods prepared through hydrothermal route // Opt. Materials, 27(3), p. 453-458 (2004).

8. P. Sudhagar, R. Sathyamoorthy, S. Chandramohan, S. Senthilarasu and Soo-Hyoung Lee, Synthesis of $\mathrm{Cd}_{1-\mathrm{x}} \mathrm{Mn}_{\mathrm{x}} \mathrm{S}$ nanoclusters by surfactant-assisted method: Structural, optical and magnetic properties // Mater. Lett. 62(15), p. 2430-2433 (2008).

9. D. Kim, M. Miyamoto, and M. Nakayama, Surface-modification effects on luminescence properties of $\mathrm{CdS}$ and $\mathrm{CdMnS}$ quantum dots prepared by a reverse-micelle method // Phys. status solidi (c) 0, No.4, p. 1233-1236 (2003).

10. M. Tanaka, Photoluminescence properties of $\mathrm{Mn}^{2+}$ doped II-VI semiconductor nanocrystals // J. Luminesc. 100, p. 163-173 (2002).

11. N.S. Norberg, G.L. Parks, G.M. Salley, D.R. Gamelin, Giant excitonic Zeeman splittings in colloidal $\mathrm{Co}^{2+}$-doped $\mathrm{ZnSe}$ quantum dots // J. Amer. Chem. Soc. 128, p. 13195-13203 (2006).

12. J.D. Bryan, D.A. Schwartz, D.R. Gamelin, The influence of dopants on the nucleation of semiconductor nanocrystals from homogeneous solution // J. Nanosci. Nanotechnol. 5, p.1472-1479 (2005).

13. S.C. Erwin, L. Zu, M.I. Haftel, A.L. Efros, T.A. Kennedy, and D.J. Norris, Doping semiconductor nanocrystals // Nature, 436/7, p. 9194 (2005).

14. C. Dridi, M. Haouari, H. Ben Ouada, A.-P. Legrand, J. Davenas, M. Bernard, J.-J. Andre, A. Haj Said, F. Mattoussi, Spectroscopic investigations on hybrid nanocomposites: CdS:Mn nanocrystals in a conjugated polymer // Mater. Sci. and Eng. C, 26, p. 415-420 (2006).
15. V.I. Fediv, A.I. Savchuk, G.Yu. Rudko, I.S. Davydenko, T.A. Savchuk, E.G. Gule, S.A. Ivanchak, Fluorescence sensing of biological tissue structures using Mn-doped CdS nanoparticles as biomarkers // Proc. SPIE, 7388, p.73880Y-1 - 73880Y-7 (2009).

16. I.I. Kocherga and A.A. Baran, Effect of nonionogenic water-soluble polymers on the electrokinetic potential of colloid particles // Theoretical and Experimental Chemistry, 12, p. 497-503 (1977).

17. A. Uhrig, A. Worner, C. Klingshirn, L. Banyai, S. Gaponenko, I. Lacis, N. Neuroth, B. Speit, K. Remitz, Nonlinear optical properties of semiconductor quantum dots // J. Cryst. Growth, 117, p. 598-602 (1992).

18. P.E. Lippens, M. Lannoo, Calculation of the band gap for small CdS and $\mathrm{ZnS}$ crystallites // Phys. Rev. $B$, 39, p. 10935-10942 (1989).

19. K. Murakoshi, H. Hosokawa, M. Saitoh, Y. Wada, T. Sakata, H. Mori, M. Satoh, S. Yanagida, Preparation of size-controlled hexagonal $\mathrm{CdS}$ nanocrystallites and the characteristics of their surface structures // J. Chem. Soc., FaradayTrans. 94, p. 579-586 (1998).

20. L.E. Brus, A simple model for the ionization potential, electron affinity, and aqueous redox potentials of small semiconductor crystallites // J. Chem. Phys. 79, p. 5566-5571 (1983).

21. J.K. Furdyna, Diluted magnetic semiconductors // J. Appl. Phys. 64, p. R29 (1988).

22. Y. Kanemitsu, A. Ishizumi, Luminescence properties of impurity-doped semiconductor nanoparticles // J. Luminesc. 119-120, p. 161-166 (2006).

23. R. Beaulac, P.I. Archer, D.R. Gamelin, Luminescence in colloidal $\mathrm{Mn}^{2+}$-doped semiconductor nanocrystals // J. Solid State Chem. 181, p. 1582-1589 (2008).

24. A.A. Bol, R. van Beek, J. Ferwerda, A. Meijerink, Temperature dependence of the luminescence of nanocrystalline CdS/ $\mathrm{Mn}^{2+} / / \mathrm{J}$. Phys. and Chem. Solids, 64, p. 247-252 (2003).

25. G. Blasse and B.C. Grabmaier, Luminescent Materials. Springer-Verlag, Berlin, 1994.

26. S. Arora, S. Sundar Manoharan, Large shift in the photoluminescent properties of $\mathrm{Mn}^{2+}$-doped nanosized CdS-ZnS solid solutions // Solid State Communs. 144, p. 319-323 (2007).

27. B. Tripathi, F. Singh, D.K. Avasthi, D. Das, Y.K. Vijay, Study of effects of $\mathrm{Mn}^{2+}$ in $\mathrm{CdS}$ nanocrystals // Physica B, 400, p. 70-76 (2007).

28. K.K. Nanda, S.N. Sahu, Photoluminescence of CdS nanocrystals: effect of ageing // Solid State Communs. 111, p. 671-674 (1999).

29. D. Kim, K. Tomihira, S. Okahara, M. Nakayama, Highly efficient preparation of size-controlled CdS quantum dots with high photoluminescence yield // J. Crystal Growth, 310, p. 4244-4247 (2008). 